\title{
Novel QoS Scheduling and Energy-saving MAC protocol for Body Sensor Networks Optimization
}

\author{
Begonya Otal \\ Centre Tecnològic de \\ Telecomunicacions de Catalunya \\ (CTTC), Barcelona, Spain \\ +34 936452918 \\ begonya.otal@cttc.es
}

\author{
Luis Alonso \\ Signal Theory \& Communications Dpt. \\ Universitat Politècnica de Catalunya \\ (UPC), Barcelona, Spain \\ +34 934137112 \\ luisg@tsc.upc.edu
}

\author{
Christos Verikoukis \\ Centre Tecnològic de \\ Telecomunicacions de Catalunya \\ (CTTC), Barcelona, Spain \\ +34 936452911 \\ cveri@cttc.es
}

\begin{abstract}
Wireless body sensor networks operate under the conflicting requirements of maintaining the desired reliability and message latency of data transmissions, while simultaneously maximizing battery-life of individual body sensors. In doing so, the characteristics of the operating environment, including physical, MAC and application layers have to be considered. The aim of this paper is the study of a novel quality-of-service fuzzy-rule based cross-layer scheduling algorithm under certain selected medical scenarios for body sensor networks optimization. To fulfill the above-mentioned requirements, not only are data packet transmissions scheduled taking the channel quality state among sensors into account, but also their packet waiting time in the accessing system and the specific body sensor applicability. Hereby we utilize an adapted distributed queuing MAC protocol that has recently been proved to be far more energy-efficient than the IEEE 802.15.4 standard for wireless sensor networks.
\end{abstract}

\section{Keywords}

Body sensor, quality-of-service, scheduling, energy-saving

\section{INTRODUCTION}

The last decade has already witnessed a rapid surge of interest in new sensing and monitoring devices for healthcare, such as the use of wearable and implanted wireless sensors for clinical applications (e.g. ECG, blood pressure, gucometer, alarms, etc). In the literature, it is already possible to find a number of publications in relation to wireless body sensor networks. Body Sensor Networks (BSNs) have the potential to greatly impact many aspects of medical care. By outfitting patients with wireless wearable or implanted vital sign sensors, collecting detailed real-time data on physiological status can be considerably simplified. However, there is a significant gap between the existing sensor network systems and the needs of medical applications. To meet these special requirements, a

Permission to make digital or hard copies of all or part of this work for personal or classroom use is granted without fee provided that copies are not made or distributed for profit or commercial advantage and that copies bear this notice and the full citation on the first page. To copy otherwise, to republish, to post on servers or to redistribute to lists, requires prior specific permission and/or a fee.

BodyNets 2008, March 13-15 Tempe, Arizona, USA

Copyright (C) 2008 ICST 978-963-9799-17-2

DOI 10.4108/ICST.BODYNETS2008.2952 successful wireless BSN design must have several unique features, which lead to a combination of interesting technical issues not found in other wireless sensor networks. In particular, medical sensor networks must support high degrees of reliability and specific message latency requirements, without endangering the overall power consumption to eliminate frequent battery replacements. That is, wireless BSNs, mainly deployed to permanently monitor human physiological parameters, must satisfy far more stringent Quality of Service (QoS) demands than those of other existing wireless sensor networks. Hence, although some intermittent packet loss due to interferences might be in some cases acceptable, persistent packet loss (due to congestion or continuous external interferences) would be problematic, placing heavy constraints on the wireless channel. Moreover, applications involving continuous healthcare monitoring require exceptionally long battery life so that the existing maintenance schedules of the monitored equipment are not compromised. Thus, the new designed solutions should provide the required low-power consumption; at the same time that rigorous QoS demands are guaranteed.

This paper follows with a brief overview of the low rate IEEE 802.15.4 (LR-WPAN) [1], which is widely considered the technology of choice for BSNs. Section 3 introduces a distributed queuing system for BSNs. The novel QoS scheduler proposal is presented in Section 4. A performance evaluation of our study and the conclusions come thereafter.

\section{PROBLEM STATEMENT}

The previous mentioned QoS provision in BSNs is tightly coupled with medium access control (MAC) protocols. The MAC layer is responsible for coordinating channel access, by avoiding collisions and scheduling data transmissions, to maximize throughput at an acceptable end-to-end packet delay and minimal energy consumption. The IEEE 802.15.4 MAC [1] is in fact intended to serve a set of applications with very low power consumption and cost requirement, and with relaxed needs for data rate and QoS. However, taking our previous analysis into account, wearable and implanted wireless sensors for clinical settings do have strict QoS requirements. Thus, to the best of our knowledge, the current IEEE 802.15.4 MAC does not comply with medical essential demands. Besides, IEEE 802.15.4 MAC saturated throughput efficiency and energy consumption performance drastically deteriorates as the number of sensors in the network increases. In the literature, both analytical models in [2] and [3] show how 802.15.4 MAC must deal with a certain 
level of data collisions, which steadily increases with the number of sensors in the network, affecting the overall system performance in saturation conditions. Therefore, IEEE 802.15.4 $\mathrm{MAC}$ is not the right choice for BSNs, since it jeopardizes BSNs scalability due to its energy-saving inefficiency and lack of QoS.

\section{SYSTEM CHARACTERIZATION}

The authors in [4] used first the Distributed Queuing Random Access Protocol (DQRAP) for local wireless communications. DQRAP divides the TDMA slot into a "reservation subslot" that is further divided into minislots, and a "data subslot". The basic idea is to concentrate user accesses in the "reservation subslot", while the "data subslot" is devoted to collision-free data transmission. DQRAP analytical model approaches the delay and throughput performance of the theoretical optimum queuing systems $\mathrm{M} / \mathrm{M} / 1$ or $\mathrm{G} / \mathrm{D} / 1$, depending on traffic source. Therefore, the system could be represented with two queues prior to two servers, thereafter named: Collision Resolution Queue (CRQ) and Data Transmission Queue (DTQ).

In our previous work in [5], we implemented Park's [2] energyconsumption analytical model and compared it to our own energy analytical modeling of a distributed queuing MAC protocol, adapted for power management issues. We showed thereby that our studied distributed queuing protocol outperforms IEEE 802.15.4 MAC in terms of saturated throughput and energy efficiency for any number of sensors. Herewith we further develop the Distributed Queuing Body Area Network (DQBAN) MAC protocol, corresponding to a system characterization specially modified for energy-efficient wireless BSNs with QoS demands. Like DQRAP, back-off periods and collisions in data packets are eliminated, thereby increasing goodput efficiency with respect to IEEE 802.15.4. In DQBAN system modeling, instead of keeping a First-Come-First-Served discipline, a QoS fuzzy-ruled scheduler is introduced as depicted in Figure 1. The scheduling technique is required to reorganize body sensors' positions in DTQ before being served in order to achieve a far more reliable system performance for medical applications. The basic idea is to take QoS criteria, channel quality and battery constraints into consideration for selecting the new ordering in DTQ, by making use of fuzzy-logic theory as a control mechanism.

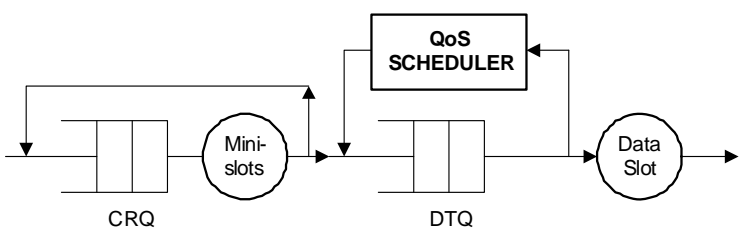

Figure 1. DQBAN System Modeling

\section{QoS FUZZY-LOGIC SCHEDULER}

The new cross-layer fuzzy-rule based scheduling algorithm pursues the idea of playing a determining role between the different physical layer states and the particular body sensors' applications. Its main goal is to optimize MAC layer performance in terms of QoS and energy consumption by applying fuzzy-logic rules into the DQBAN system modeling. The scheduling system depends on three input variables derived from each body sensor's precise applicability and the interaction with changeable and independent environmental conditions (i.e. wireless channel, system load) in order to decide the new order in DTQ. Bearing in mind the dynamic and unpredictable constraints of our system, we make use of fuzzy-logic theory for the sake of simplicity in the algorithm implementation.

\subsection{Fuzzifier and Defuzzifier}

In general, a Fuzzy Logic System (FLS) is a nonlinear mapping of an input data vector into a scalar output. Fuzzy set theory establishes the specifics of the nonlinear mapping [6]. Figure 2 depicts a FLS that is widely used in fuzzy logic controllers. It contains four components: fuzzifier, fuzzzy rules, inference engine, and defuzzifier

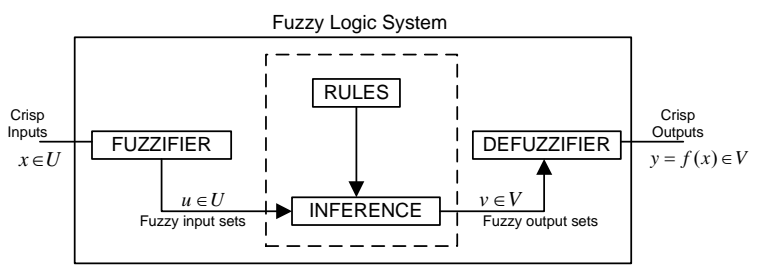

Figure 2. Fuzzy Logic System

The QoS fuzzy-logic scheduler mechanism is a nonlinear system and can be interpreted as a fuzzy-logic controller implemented in every body sensor (i). The FLS is fulfilled with three sensordependant time-variant input variables from diverse nature: i) Signal-to-Noise Ratio - SNR $(i, t)$ - derived at the reception of a feedback frame [5]; ii) Waiting Time in the system - WT(i,t) calculated from an inherent clock, and iii) residual Battery Life $B L(i, t)$ - derived from an inner hardware memory. However, in order to facilitate the implementation design at the entrance of the fuzzy-logic scheduling system, we use normalized values with respect to each body sensor specific constraints: $S N R_{\min }(i)$, derived from the Bit-Error-Rate $(B E R(i)) ; W T_{\max }(i)$ and $B L_{\min }(i)$, which consider application-related latency and minimal battery lifetime requirements. Thus, at the entrance of the fuzzifier there will be the following normalized input crisp variables:

$$
\begin{aligned}
& {[\operatorname{SNR}(i, t)]=S N R(i, t)-S N R_{\min }(i)} \\
& {[W T(i, t)]=W T(i, t)-W T_{\text {max }}(i)} \\
& {[B L(i, t)]=B L(i, t)-B L_{\text {min }}(i)}
\end{aligned}
$$

The input normalized crisp variables in the fuzzifier are hereby identified to the fuzzy sets as following; the input linguistic terms \{dangerous, poor, superior\} will constitute the antecedents of the linguistic rules for the associated input fuzzy variable SNR. The set of linguistic values \{acceptable, boundary, excessive $\}$ and \{critical, balanced, substantial\} are associated to the input fuzzy variables WT and BL, respectively. The output fuzzy variable DTQp, at the entrance of the defuzzifier, has been associated to the fuzzy set \{delay, onschedule, forward $\}$, which form the consequents of our fuzzy rules. A body sensor decision can be to delay its transmission to a future frame, to keep its current position in DTQ by indicating onshedule, or to ask for a prior position in DTQ depicted by forward. Figure 3 portrays an illustrative example of the membership functions used in our fuzzy-logic system for all the same sort of antecedents and consequent. 
The representation of linguistic 2 is an isosceles triangle and the value of $X_{b}$ has been adjusted by computer simulations to the known values of $S N R_{\text {min }}(i), W T_{\max }(i)$ and $B L_{\text {min }}(i)$, for our studied scenario thereafter. We chose triangular functions for its simple expression at the output of the defuzzifier by using the height deffuzzifer as explained in [6]. The output crisp variable $D T Q p(i, t)$ will represent the body sensor (i) decision at the time $(t)$ with respect to its current position in DTQ.

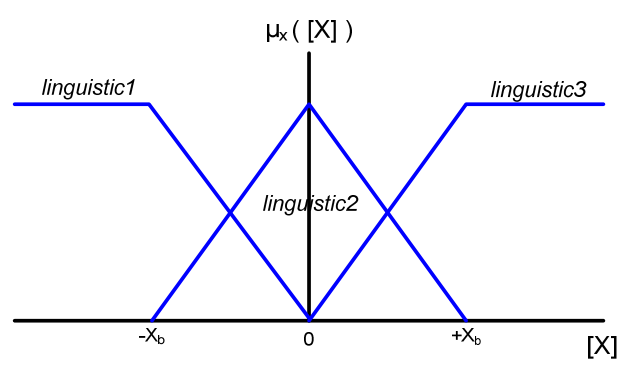

Figure 3. Membership function example

\subsection{Fuzzy-logic Rules}

Since the fuzzy input variables SNR, WT, and BL have each three different states, the total number of possible ordered pairs of these states is 27 . For each of these, we have to determine an appropriate state of the output fuzzy variable DTQp. A convenient way of defining all required rules is through a decision table, which is also called a fuzzy association memory (FAM) bank matrix. It consists hereby of $27(3 \times 3 \times 3)$ rules and is constructed for DTQp decision as shown in Table 1.

Table 1. Fuzzy-logic rules for DTQp decision

\begin{tabular}{|c|c|c|c|c|}
\hline \multirow{2}{*}{ WT } & \multicolumn{3}{|c|}{ SNR } & \multirow{2}{*}{ BL } \\
\cline { 2 - 5 } & dangerous & poor & superior & substantial \\
\hline acceptable & delay & delay & onschedule & subs \\
\hline acceptable & delay & delay & onschedule & balanced \\
\hline acceptable & delay & delay & delay & critical \\
\hline boundary & delay & onschedule & onschedule & substantial \\
\hline boundary & delay & onschedule & onschedule & balanced \\
\hline boundary & forward & forward & forward & critical \\
\hline excessive & forward & forward & forward & substantial \\
\hline excessive & forward & forward & forward & balanced \\
\hline excessive & forward & forward & forward & critical \\
\hline
\end{tabular}

The result of the fuzzy inference engine is that a set of linguistic values of the fuzzy variables SNR, WT, BL is assigned to a linguistic output value of the fuzzy variable DTQp as,

$$
\begin{aligned}
& \mathrm{R}^{(1)}: \text { IF } S N R_{1} \text { is } a_{1}^{l} \text { and } W T_{1} \text { is } a_{2}^{l} \text { and } B L_{1} \text { is } a_{3}^{l} \\
& \text { THEN DTQp is } c^{l}
\end{aligned}
$$

$a^{l}$ and $c^{l}$ are the fuzzy sets corresponding to the antecedent and consequent of the $1^{\text {th }}$-rule.

\section{PERFORMANCE EVALUATION}

The DQBAN protocol is hereby employed in a star-base topology where a Body Area Network (BAN) coordinator broadcasts feedback information about the situation of the queues at the end of each frame [5]. This scenario matches most monitoring applications, since body sensors send periodical data to a single monitor or data storage device.
Nevertheless, DQBAN is distributed as well, because every body sensor independently actualizes its position and the status of the two "virtual" common queues in the system following strategic rules. In practice, the queuing system implementation is simply run through four integers in each sensor; two common numbers shared among all body sensors representing the total amount of occupied positions in CRQ and DTQ, and two other different integers, $\mathrm{pRQ}_{\mathrm{i}}$ and $\mathrm{pTQ}_{\mathrm{i}}$, which exclusively indicate the body sensor position in each queue. Additionally, a fuzzylogic controller is used by every body sensor as explained before.

\subsection{Case Study}

The performance of the proposed techniques is evaluated in a BAN with 4 medical sensors and $N$ pervasive body sensors. A 12-lead ECG body sensor, a Blood Analysis body sensor, a Supervisory body sensor, and an Alarm body sensor are characterized with a constant traffic distribution at different loads, specific BER and latency values as described in [7]. Further, we add $N$ pervasive always-active body sensors following a Poisson distribution with $65 \mathrm{~ms}$ of mean packet inter-arrival time, in order to assess our scalability system performance. Hence we evaluate a heterogeneous BSN system performance considering diverse traffic modeling and sensordependant QoS specifications. The battery lifetime is the same for each sensor in our current simulations.

For simplicity, we have based our calculations on the average path loss model taken from [1] within a hospital distance room $(\mathrm{d}<8 \mathrm{~m})$. The time-variant received signal model includes Additive White Gaussian Noise (AWGN) and the effect of lognormal shadowing with a standard deviation $\sigma=30 \mathrm{~dB}$ for our BSN simulation environment. The conditions of an AWGN channel are reproduced to obtain $\operatorname{SNR}(i, t)$ as a function of the received power, and $S N R_{\min }(i)$ is derived from each sensor specific BER for a O-QPSK modulation as defined in [8].

The assumption of an AWGN channel is valid as long as the channel is coherent during the transmission of a packet (slow fading). We use IEEE 802.15.4 MAC reference parameters with the maximum payload packet size of 118 bytes transmitted at the unique rate of $250 \mathrm{Kbps}$. Therefore, a packet transmission takes roughly $4.27 \mathrm{~ms}$, which is smaller than the coherence time encountered in the $2.4 \mathrm{GHz}$ band without mobility issues [9] (i.e. $31.25 \mathrm{~ms}$ if velocity of scatters is $1 \mathrm{~m} / \mathrm{s}$ ). Note that each body sensor waiting time in the system and its residual battery lifetime are calculated following our work in [5].

\subsection{Simulation Results}

By means of MATLAB computer simulations, the metrics used herewith to evaluate the DQBAN system performance in terms of QoS are the packet loss probability and the mean packet endto-end delay as the number of pervasive sensors increases from 5 to 11 (i.e. from 9 to 15 body sensors in total). Each curve represents the results for each medical sensor separately (i.e. ECG, Blood Analysis, Supervisory and Alarm) and the average calculated for the total amount of Pervasive sensors after long iterating the system performance.

We compute first the probability of packet success $\boldsymbol{p}_{\text {suc }}$ at the time of transmitting within the wireless channel as defined in Table 2, where $\theta=S N R(i, t)$ and $\delta=S N R_{\min }(i)$; Table 2 is the same for each body sensor, though it depends on its $\operatorname{SNR}(i, t)$. 
Table 2. Probability of success

\begin{tabular}{c|c|} 
& $\boldsymbol{p}_{\text {suc }}$ \\
\hline $2(\delta)<\theta$ & $\mathbf{0 . 8}$ \\
\hline$\delta<\theta<1.99(\delta)$ & $\mathbf{0 . 5}$ \\
\hline $0.8(\delta)<\theta<\delta$ & $\mathbf{0 . 1}$ \\
\hline$\theta<(0.79) \delta$ & $\mathbf{0 . 0 0 1}$ \\
\hline
\end{tabular}

Hence the packet loss probability is the ratio of packets that could not be transmitted successfully, taking the probability of success in the wireless channel into account, the packet timeout due to latency limits, and battery limitations, specified by each body sensor. Our simulation results for the packet loss probability without and with scheduler are portrayed in Figure 4 and Figure 5 respectively. It is shown how the fact of using the QoS scheduler with the strategic fuzzy-logic rules improves from $15 \%$ to $25 \%$ the system performance in terms of reliability. Packet loss probability decreases with the number of sensors in Figure 5, thanks to the QoS scheduler, since it is always possible to find a body sensor in DTQ with an optimal wireless link.

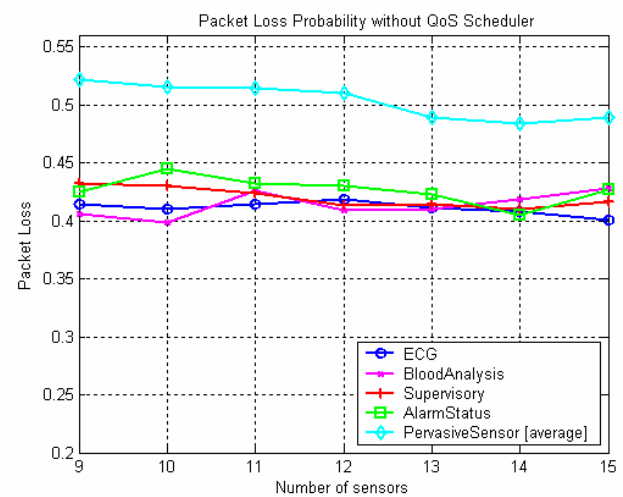

Figure 4. Packet-loss probability without QoS Scheduler

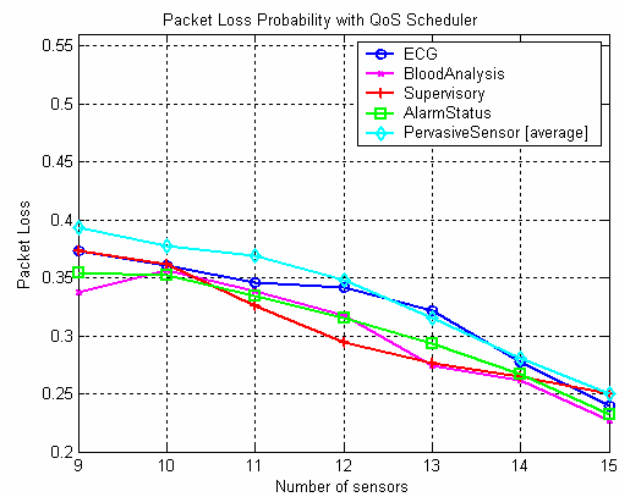

Figure 5. Packet-loss probability with QoS Scheduler

Further, we compute the mean end-to-end packet delay of DQBAN modeling based on our work in [5] and prove that the QoS scheduling algorithm does not affect the delay system performance. Figure 6 depicts how the mean end-to-end packet delay increases with the number of sensors, though all body sensors satisfy their latency limits as previously specified in our studied scenario. Energy consumption is also minimized as shown in [5].

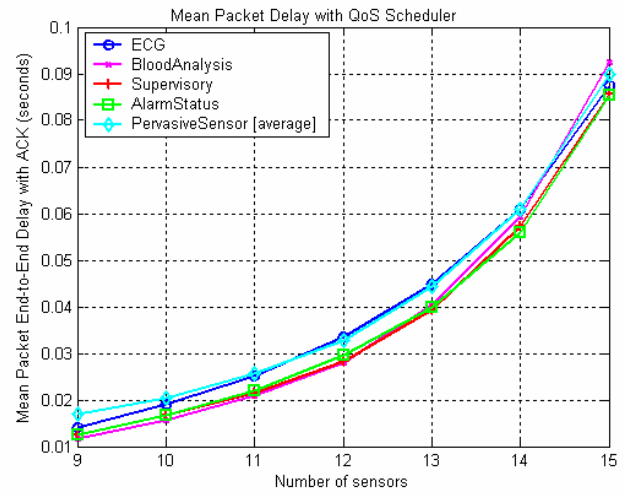

Figure 6. Mean end-to-end delay

\section{CONCLUSIONS}

In this paper, we introduced a novel QoS cross-layer scheduling mechanism based on fuzzy-logic rules that operates on top of an energy-saving distributed queuing MAC protocol for body sensor networks. Depending on each body sensor application, the new system commitment is to guarantee that all its packets are served with a specific Bit-Error-Rate (BER) and within particular latency limits, without endangering battery life. We evaluated the system under certain medical scenarios and verified the correct operation of the proposal by computer simulations. Our results show that the system performance with the QoS scheduler is from $15 \%$ to $25 \%$ more reliable than without the scheduler. Further, the mean end-to-end delay of the new modeling does not affect the overall system results.

\section{REFERENCES}

[1] "IEEE 802.15.4, MAC and PHY Specification for LRWPANs", IEEE, October 12003.

[2] T.R. Park, et al. "Throughput and Energy Consumption Analysis of IEEE 802.15.4 slotted CSMA/CA", Electronic Letters, 1st September 2005, Vol. 41, No.18.

[3] S. Pollin et al. "Performance Analysis of Slotted IEEE 802.15.4 Medium Access Layer", Technical Report, DAWN Project, Sep. 2005.

[4] H.J. Lin and G. Campbell, "Using DQRAP for local wireless communications", in Proc. of Wireless '93, Calgary, Canada, July 1993, pp. 625-635.

[5] B. Otal, C. Verikoukis, L. Alonso, "Efficient Power Management based on a Distributed Queuing MAC for Wireless Sensor Networks", in Proc. of IEEE VTC'07Spring, Dublin, Irland, 22-25 April 2007.

[6] J.M. Mendel, "Fuzzy Logic Systems for Engineering: A Tutorial", in Proceedings of the IEEE, Vol. 83, pp. 345377, March 1995.

[7] N. Golmie, D. Cypher, O. Rebala, "Performance evaluation of low rate WPANs for medical applications", in Proc. of IEEE MILCOM 2004, pp.927- 933. Vol. 2, November 2004.

[8] J.G. Proakis, Digital Communications, McGraw Hill, 2001.

[9] S. Thoen, et al. "Modeling the Channel Time-Variance for Fixed Wireless Communications", IEEE Communications Letters, Vol. 6, No.8, August 2002. 